\title{
On Effect of Planar Scaling on Microstrip Patch Antenna Performance
}

\author{
Ahmad Esmaeilkhah ${ }^{1}$, Changiz Ghobadi ${ }^{1}$, Javad Nourinia ${ }^{1}$ and Maryam Majidzadeh ${ }^{2}$ \\ ${ }^{1}$ Electrical Engineering Department, Urmia University, Urmia, Iran. \\ ${ }^{2}$ Electrical and Computer Engineering, Urmia Girls Faculty, West Azarbaijan branch, Technical and Vocational University \\ (TVU), Urmia, Iran \\ *Ahmad Esmaeilkhah, E-mail: a.esmaelkhah@urmia.ac.ir
}

\begin{abstract}
This paper aims at investigating the effect of planar scaling on microstrip patch antenna performance. To this end, nine antennas with different sizes are fabricated on the same FR4 substrate with thickness of $1 \mathrm{~mm}$ with different scale factors. Results indicate some deviations between the expected and obtained simulated and measured resonant frequency which could be mainly due to the truncation error effect, frequency dependent properties of selected substrate and the SMA connector effect in the scaling process. All the influential factors are studied in detail through the paper. Also, the theoretical study of findings provides some mathematical models which help to predict the deviation of measured and simulated resonant frequency in respect with expected values. Detailed discussion on the scaling process and its effect on the antenna performance would be presented.
\end{abstract}

\section{Introduction}

The tendency and ability to solve difficult problems with the aim of establishing new techniques to invent desirable processes is one of the most brilliant human capabilities. The mathematical model of dynamic properties of coupled electric and magnetic fields, the Maxwell Equations (ME), is an excellent example of such an achievement [1]. These equations are valid, regardless of the size and complexity of the structure. Also their scale-invariable property, which was investigated and proved by Hamdan [2], has attracted considerable attention in different applications, such as Radar Cross Section (RCS) measurement [3]. Historical review of scaling and its applications in electromagnetism reveals the great devoted attempt on scaling of the original structure into a smaller one $[4,5]$. However, the advent of some new concepts, such as Nantenna [6] and Rectenna [7], has called the need of new requirement in scaling technique which needs to reverse the scaling procedure, that is, to scale up a Nano-sized structure for initial prototyping, measurement and tuning.

Clearly, the scaling is an interesting process, due to the fact that, it makes the measurement process more easy and possible. As one of the first attempts, the scaling of a $2.4 \mathrm{GHz}$ rectenna by NASA could be found in [8]. Similar to any other mathematical models, the MEs do not dealt with limitations of real world applications and require some especial considerations while the predictions are carried out.
These limitations are inherent nonlinear or frequency dependent properties of materials [9], mechanical tolerances in manufacturing processes [10], the method and precision of measurement techniques [11] and expressing methods [12], which should be investigated for any accurate predictions.

As a prerequisite to the main problem, the findings of most brilliant scholars such as Galileo Galilei which described as Galilean Transformation (GT) [13, 14] is explored. Any Newtonian Equations are invariant under GT [15]. This phenomena means that the measurement of location, speed, acceleration, and etc. are identical in any random frame, or equally any arbitrary coordination system, regardless of its linear speed. This great observation was valid until the emergence of MEs in 1865 [1]. The four constituent equations of introduced mathematical model were shown good convergence into experimental results, but were not valid after applying GT [15]. So the MEs are not invariant under GT. For example, applying GT to Gauss' law yields in unbounded increase of electron density during the time pass, which is in contradiction with law of charge conservation [14].

Poincare's investigations on Lorentz works yielded the establishment of Lorentz Transformation, or simply the LT, which tied the space and the time using Lorentz Factor [16]. This means that in any set of LT-invariant equations the time and space dependent variables could be scaled using appropriate considerations.

The newly introduced space-time concept, mutually, redefined the imaginations about coordination systems. Einstein's special relativity finalized the investigations by speculation of constancy of speed of light for any random frame of reference [17]. The well-known and ancient 3D coordination system should dance harmoniously with Time to be adapt the empirical results with mathematical models of electromagnetism. This caused the MEs to be invariant under LT, a great discovery which was less discussed thoroughly. Scalability of electromagnetic structures, as a necessary consequence of MEs' invariability under LT, was first studied and formulated by Sinclair, by defining four dimensionless scaling parameters, $\alpha, \beta, p$ and $\gamma$ and applying some assumptions [15]. The formulated results were categorized into "Absolute Model" and "Practical Geometrical Model" and are partially still in use. The concept was again surveyed meticulously by Hamdan and his collogues [2].

Some specific concepts or applications of scalability of 
microstrip structures were studied before [8, 18, 19 and 24]. To complete an accurate insight into the issue and to generate useful estimations on effect various participating but unavoidable parameters on predictions, this paper compares simulation, prediction and measurement results of nine antennas which all of their dimensions are scaled except of substrate thickness.

\section{Methods and Materials}

To ensure the appropriate scaling of the MEs and the electromagnetic structure, some theoretical and technical considerations are applied. These considerations also control the level of unwanted errors during numerical simulation of the proposed structures. It is worth noting that the study is carried out for a sample antenna. To keep the generality of the study, the exact value of parameters are not a matter of interest.

\subsection{Computer Aided Design Simulation}

A simple rectangular microstrip-fed patch antenna, shown in Figure. 1, is selected as the sample antenna. The arbitrary size of the antenna and consequently its non-tuned features ensures the generality of study. The antenna is printed on FR4 substrate with $\varepsilon_{r}=4.4$ and thickness of $1 \mathrm{~mm}$. Length and width of the antenna are $159 \mathrm{~mm}$ and $92 \mathrm{~mm}$ respectively. All the extracted results are obtained using Ansoft High Frequency Structural Simulator (HFSS) V13.0.

To ensure the accuracy of simulation and its conformity to required considerations [4], it should be mentioned that in all of the simulations the bandwidth was set to be less than $0.01 \%$ of simulation frequency, or $f_{s}$, and was achieved by iterative fine tuning of the $f_{s}$ and simulation bandwidth. This ensures that appropriate sizing of the meshes during the simulation process. Likewise, the calculated resonant frequency of antenna or $f_{r}$ and $f_{s}$ were set to be adjacent and the maximum achieved difference is $0.13 \%$ of $f_{s}$. The simulated metallic layers of patch antenna were considered to be with a thickness of $0.35 \mu \mathrm{m}$ and to be as rough as $0.017 \mu \mathrm{m}$ [22]. Moreover, the final antennas are not metallized during their production and will be remained unchanged for various scale-factors, or simply $S_{F}$. Hence, the metallic layers' conductivity was set to be equal to copper's conductivity. The associated air-box and the wave-port of the simulation were scaled automatically using mathematical formulation in simulation coordination system.

With the aim of exploring the scaling effect on antenna performance, nine antennas, including the initial one, with equal shape and various sizes are designed, manufactures and some of their essential parameters are studied. As all the antennas have similar physical shapes, and only the sizes are different, the simulation results would directly reveal the scaling effect on antenna performance. It is worth noting that different scaling processes could be envisages. As an instance, the effect of substrate thickness scaling was investigated thoroughly in [18], it was excluded to be scaled and the $2 \mathrm{D}$-scaling is focused.

It is worth mentioning that some of actual values and parameters are not scalable due to technological or practical limitations. Inability of scaling the surface roughness, which will directly affect the surface resistance of metallic parts at higher frequency, was firstly seen and reported in RCS measurement applications [21]. Moreover, chemical or LASER etch techniques will smoothen the surface as much as possible, but the actual degree of smoothness is not under control and is useless in precise scaling processes [22].

As another one, the electrical conductivity of scaled metallic parts is required to be squared with respect to scaling factor [4]. The electrical conductivity is an inherent parameter of materials and mostly could not be controlled, especially while not using superconducting metals. The aforementioned parameters, which could not be scaled, are the same for all the nine antennas and their effect could be suitably neglected.

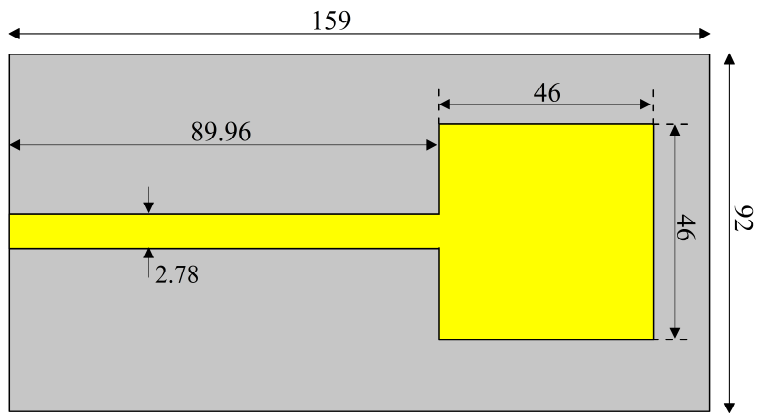

Figure. 1. The dimensions of $A_{1}$ antenna in millimeters.

\section{2. $S F S$}

The SFs could be selected arbitrarily, as it is a dimensionless real number. Herein, the SFs are chosen based on available thicknesses of FR4 substrate, defining nine scales factors, ranging from 1 to 0.078125 , as reported in Table 1. As the paper has focused on the "Planar Scaling", the substrate thickness is $1 \mathrm{~mm}$ (z-axis) in simulation and for fabricated antennas. The values of "Available Substrate Thicknesses" have been used to calculate the Scaling-factor for $\mathrm{x}$ - and $\mathrm{y}$ - axis. This means all of the antennas are simulated for FR4 substrate with constant thickness of $1 \mathrm{~mm}$ and fabricated on it (constant $\mathrm{z}$ ), but with scaled planar size (scaled $\mathrm{x}$ and $\mathrm{y}$ ). The effect of substrate thickness on resonance frequency of microstrip antenna includes some "erratic" results for thicknesses greater than $0.02 \lambda_{0}$ and inconsistency in prediction of resonant impedance [18]. For further investigation variable thickness of substrate is excluded to be scaled and the 2D-scaling is focused.

The constant substrate thickness violates the scaling principles [4]; so the results will not follow the predictions as expected and some drifts would be measured. The initial size of $A_{1}$ is of high importance due to the limitations of available PCB production technologies. In fact, the initial values should be selected so that the smallest antenna could be manufactured easily using the available manufacturing technologies.

\subsection{Truncation Error Considerations}

The available computational resources, such as memory or processing capabilities, limits the number of handle-able 
digits of Real numbers. The eliminated part causes small errors which become more and more considerable in massive numerical calculations. The effect of Truncation Error during numerical calculations has been investigated previously and governing formulation for upper limit of truncation error, namely $E_{T}$, has been reported [12]. Herein the unbalanced number of preserved digits for measurement, 10 digits, and simulation, 16 digits, was chosen to lower the $E_{T}$ as low as $0.01 \%$ of non-truncated value. This is also the maximum difference of measured and simulated values which could be caused by truncation processes.

Table 1. The scale-factor and dimensional scaling properties of simulated antennas.

\begin{tabular}{c|c|c|c|c}
\hline $\begin{array}{c}\text { Available } \\
\text { Substrate } \\
\text { Thicknesses } \\
(\mathbf{m m})\end{array}$ & $\begin{array}{c}\text { Substrate } \\
\text { Thickness } \\
\text { of } \\
\text { Simulated } \\
\text { Antennas } \\
(\mathbf{m m})\end{array}$ & $\begin{array}{c}\text { Scale } \\
\text { Factor }\end{array}$ & $\begin{array}{c}\text { Simulated } \\
\text { Antennas Size } \\
(\mathbf{m m})\end{array}$ & $\begin{array}{c}\text { Ant. } \\
\text { Codes }\end{array}$ \\
\hline \hline 3.2 & 1 & 1 & $159 \times 92$ & $\mathrm{~A}_{1}$ \\
\hline 2.4 & 1 & 0.75 & $119.25 \times 63.25$ & $\mathrm{~A}_{2}$ \\
\hline 2.2 & 1 & 0.6875 & $109.312 \times 63.25$ & $\mathrm{~A}_{3}$ \\
\hline 2 & 1 & 0.625 & $99.375 \times 57.5$ & $\mathrm{~A}_{4}$ \\
\hline 1.6 & 1 & 0.5 & $79.5 \times 46$ & $\mathrm{~A}_{5}$ \\
\hline 1 & 1 & 0.3125 & $49.6875 \times 28.75$ & $\mathrm{C}_{5}$ \\
\hline 0.8 & 1 & 0.25 & $39.75 \times 23$ & $\mathrm{~B}_{6}$ \\
\hline 0.5 & 1 & 0.15625 & $24.843 \times 14.375$ & $\mathrm{~A}_{6}$ \\
\hline 0.25 & 1 & 0.078125 & $12.422 \times 7.188$ & $\mathrm{~A}_{7}$ \\
\hline
\end{tabular}

\subsection{Antenna Manufacturing}

All the nine antennas with the aforementioned $S_{F} \mathrm{~S}$, as listed in Table 1, are fabricated on 1mm FR4 substrate and are shown in Figure. 2. Similar Computer Numerical Control router is adopted in cutting process of all the antennas. It is a well-studied fact that dielectric properties of FR4 substrate are widely violated for various FR4 sheets manufacturer [23]. To eliminate the probable introduced error due to use of sheets from different manufacturers, the antennas are printed on FR4 sheets from single vendor and from a single batch of production.

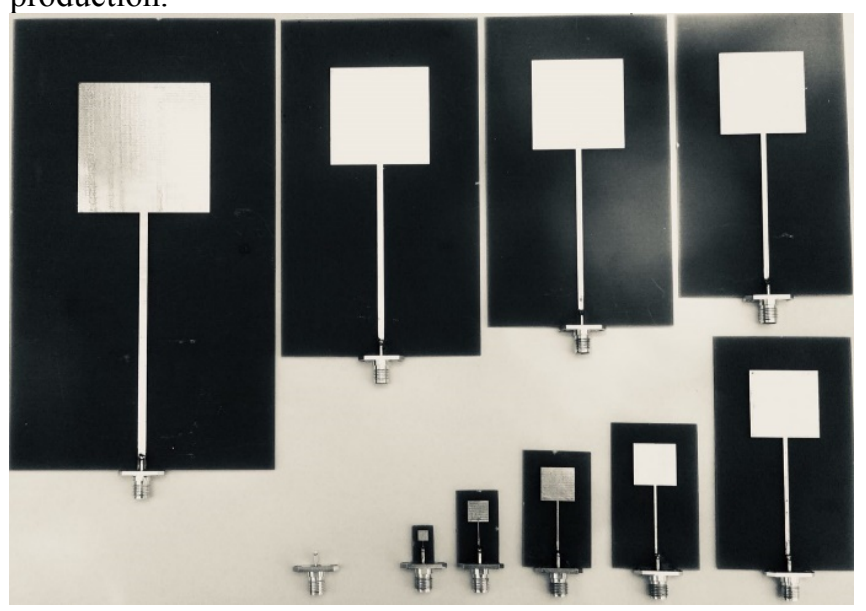

Figure. 2. Fabricated antennas with mounted SMA connector.
For measurement purpose and as the standard electrical connectors are not scalable, SMA connectors of minimum size from a known manufacturer are selected and soldered to the aforementioned nine antennas carefully.

\section{Results and Discussion}

To shed light on the scaled microstrip-fed antennas' performance, the fabricated antennas are measured in antenna and microwave laboratory. Before measurement, the antennas are cleansed using isopropyl alcohol (IPA) to remove any dust, soldering debris and any unwanted materials such as soldering oil. The first resonant frequency and complex value of $\mathrm{S}_{11}$ are measured. The obtained data are sorted, post processed and compared with the simulated ones. Finally four set of information are extracted and studied as follows:

\section{1. $S_{11}$ Variation}

$\mathrm{S}_{11}$ curves for the aforementioned nine antennas in $400 \mathrm{MHz}$ span around their resonant frequency are shown in Figure. 3. It is clearly seen that variation of $S_{F}$, causes significant variation in resonant frequency of antennas. The A 7 antenna, despite its fluctuating behavior, is the most matched one as its $\mathrm{S}_{11}$ 's magnitude in $36 \%$ less than A1's at their resonant frequency. It can be considered as direct effect of reduction in $S_{F}$, but as it will be shown later in this paper, this phenomena is due to unwanted effect of selected electrical connection. As can be seen, reduction of $S_{F}$ results in fluctuations increase. This is the side effect of using SMA connector which was not scaled down during scaling process.

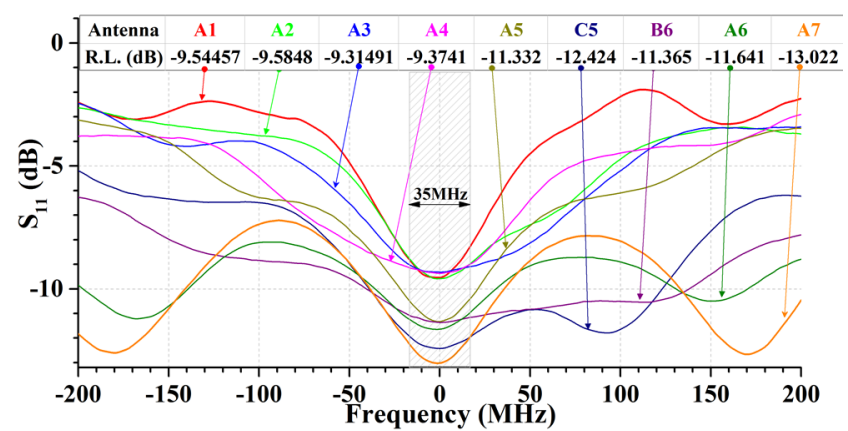

Figure. 3. The variation of $S_{11}$ of various antennas in $400 \mathrm{MHz}$ span around their resonant frequency. The hashed are in center of plot is the equal fractional bandwidth of $A_{1}$ in respect to $400 \mathrm{MHz}$ for $\mathrm{A}_{7}$.

\subsection{Resonance Frequency}

The expected, simulated and measured values of resonant frequency are shown in Figure. 4. There are measurable deviation between measured and simulated values of resonance frequency and the predicted ones. Also this deviation is $S_{F}$ dependent; as it is illustrated clearly in Figure. 5 , for $\mathrm{C}_{5}$, and $\mathrm{B}_{6}$. The value of resonant frequency which obtained from simulation and measurement for $A_{1}$ is considered to be the initial expected value of resonant frequency, or $f_{e}$. The expected resonant frequency for various $S_{F} \mathrm{~S}$, is calculated as:

$$
f_{r-\exp e c t e d}=f_{e} /\left(S_{f}\right)^{p}
$$


While $p=1$, a direct consequence of scale-invariability of Maxwell equations [4]. As can be seen, both curves, which deviate from expected value, are converging. Deviations are exponentially changing and seem to increase unbounded. Normalized deviations as large as $5.7 \%$ and $8.4 \%$ for measured and simulated resonant frequency in the case of $\mathrm{A}_{6}$ are measureable and indispensable.

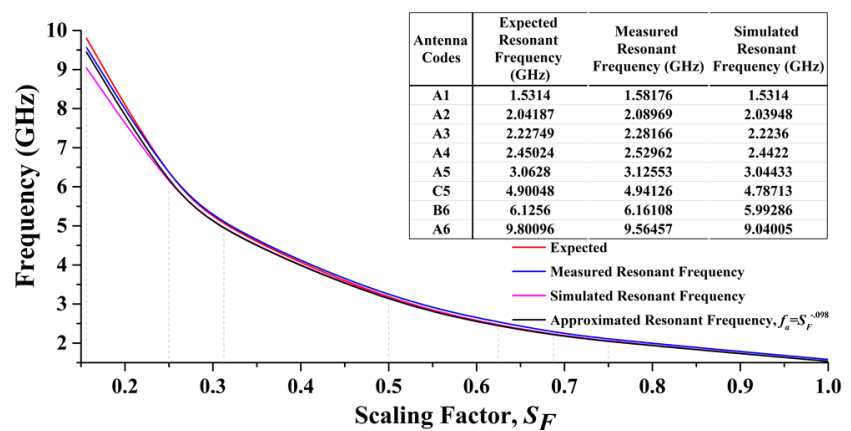

Figure. 4. The simulated, measured and expected resonant frequency of antennas. The "Approximated Resonant Frequency" models the behavior of "Measured Resonant Frequency" curve mathematically and is slightly different form theoretical predictions, $p=0.98$ instead of $p=1$.

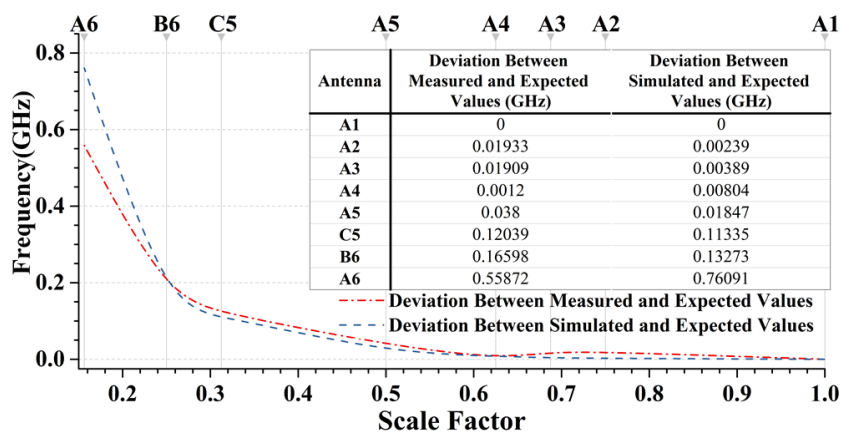

Figure. 5. Deviation of measured and simulated resonant frequency in respect with the expected resonant frequency of antennas.

\subsection{S11 Magnitude Deviation}

Based on the results obtained in [4], the final scaling coefficient for power levels is $\left(S_{F}\right)^{-2}$. The definition of $S_{11}$, as logarithmic division of reflected and incident power, will remain unchanged during the scaling process. Hence, it is expected to have the measured $\mathrm{S}_{11}$ of $\mathrm{A}_{1}$ unchanged during scaling process. Measured and simulated values of $\mathrm{S}_{11}$ magnitudes at correspondent resonant frequency of each antenna are tabulated and their respective curves are illustrated in Figure. 6.

As described in section 2.1, the initial size of the antenna, for $A_{1}$, was selected arbitrarily. So the exact value of its $S_{11}$ is not of interest so far. The simulated results show meaningful, predictable and smooth decrease in $\mathrm{S}_{11}$ magnitude. Also the measured values follow the decaying behavior of simulated ones, but with some fluctuations. Finally the measured value drifted about $-36.4 \%$, means that the antennas with lower SFs exhibit better matching. The small fluctuation is mainly because of unwanted effect of unscaled SMA connector that will be discussed in detail in next section.

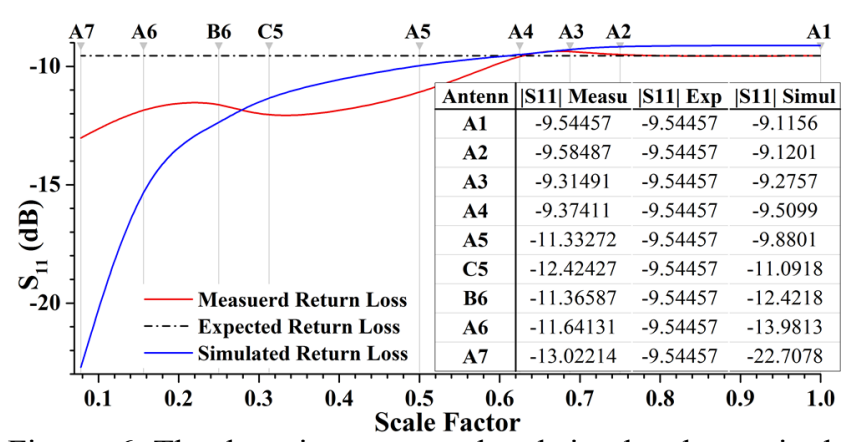

Figure. 6. The decaying measured and simulated magnitude of $\mathrm{S}_{11}$ for various antennas (with soldered SMA connector) as function of scale-factor.

\subsection{The SMA Connector}

Being the same for all of the antennas, the effect of SMA connector is also supposed to be the same. But the no-load $\mathrm{S}_{11}$ curve of the connector reveals its unpredictable effect on overall performance of antennas. The mentioned curves beside the values of that at resonant frequency of proposed antennas are illustrated in Figure. 7.

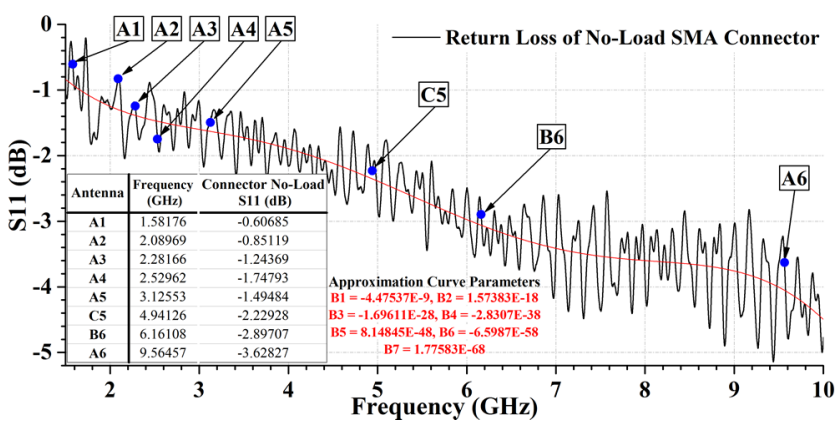

Figure. 7. The no-load $\mathrm{S}_{11}$ of SMA connector (without microstrip antenna). The position of resonant frequency of various antennas were shown using bold blue dots. Also the associated parameters of deployed Logistic Approximation curve (red solid line) were provided too.

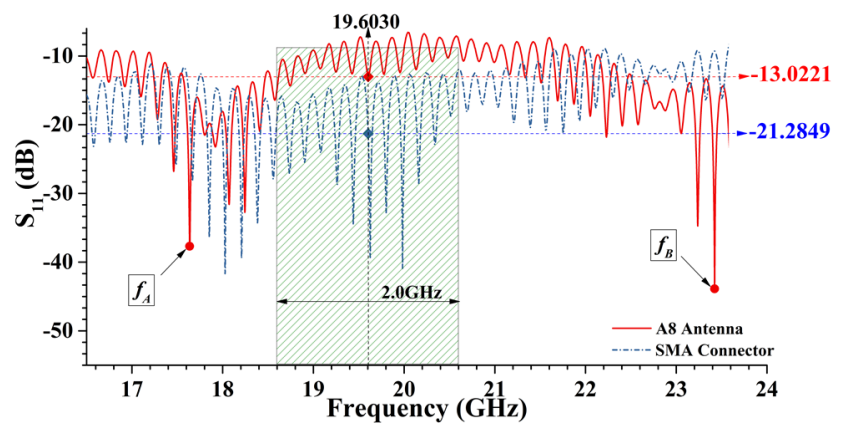

Figure. 8. The no-load $\mathrm{S}_{11}$ of SMA connector and $\mathrm{S}_{11}$ magnitude of $A_{7}$ antenna. The hashed area represents the expected window which $\mathrm{A}_{7}$ resonant frequency must be found.

The $\mathrm{S}_{11}$ curve of SMA connector decreases as the frequency increases from 1.5 to $10 \mathrm{GHz}$. Especially at 
frequencies above $15 \mathrm{GHz}$, the connector starts to radiate very efficiently and affects the overall performance of $A_{6}$ antenna as shown in Figure. 8. The fluctuating behaviors of SMA connector's performance and $\mathrm{S}_{11}$ of $\mathrm{A}_{7}$ antenna at frequencies as high as 16.5 to $24 \mathrm{GHz}$ are also depicted in Figure. 8. Based on simulation results, the resonant frequency of $\mathrm{A}_{7}$ was predicted to be around $19.6030 \mathrm{GHz}$. Due to previously described errors and tolerances, the proposed antenna's resonant frequency should be found in $\sim 2.0 \mathrm{GHz}$ span around the expected frequency, as illustrated with hashed area in Figure. 8 .

As seen, the SMA connector shows to be efficiently matched at this frequency range, even in the absence of its radiative structure. Also $\mathrm{S}_{11}$ curve fluctuates harmoniously in about every $180 \mathrm{MHz}$. This results in eleven local minimum in connector's $\mathrm{S}_{11}$ curve within $2.0 \mathrm{GHz}$ symmetrical span around expected frequency. It is worth noting that the connector's $\mathrm{S}_{11}$ magnitude at the expected resonant frequency of $A_{7}$ is at least 2.5 to 7 times of that of the other antennas. Also $f_{A}$ and $f_{B}$ are retarded and advanced resonant frequencies of $\mathrm{A}_{7}$, respectively 2 and $3.8 \mathrm{GHz}$ away of expected resonant frequency. There are no clear evidence showing they are the scaled versions of resonant frequency of other antennas. This is the reason of absence of $A_{7}$ 's performance characteristics in some of illustrated figures.

\section{The Theoretical Interpretations and Mathematical Models}

Scaling, as manipulation of coordination system of an electromagnetic structure, showed some sort of deviations between the numerical simulation results and real-world measurement values in respect with their correspondent theoretically calculated expected values. As the shape of antennas were scaled precisely, the calculated deviation, i.e. in Figure 4, is independent of shape of antenna. This deviation show an exponential-like behavior which is clearly $S_{F^{-}}$ dependent. While the frequency-dependent properties of FR4 substrate varies smoothly, the deviation between calculated and measured resonant frequencies could be calculated as:

$\Delta f_{E: M}=0.00988+29.48002 \mathrm{e}^{-\mathrm{u}\left(\mathrm{S}_{\mathrm{F}}\right)}$

While

$$
u\left(S_{F}\right)=\left(84.57412 S_{F}\right)^{0.53584}
$$

While the $S_{F}$ is the dimensionless Scaling-factor of antenna. On the other hand, the deviation between simulated and measured values for resonant frequency could be modeled as:

$$
\begin{aligned}
& \Delta f_{E: S}=398.59679-398.59386 \mathrm{e}^{-\mathrm{u}\left(\mathrm{S}_{\mathrm{F}}\right)} \\
& \text { While } \\
& u\left(S_{F}\right)=\left(40.93458 S_{F}\right)^{-3.37676}
\end{aligned}
$$

As seen in Figure 9, these approximations are useful to calculate the upper- and lower-limit of probable deviation in resonant frequency of the scaled antennas. Also, at $S_{F-e} \approx 0.21$ the deviations are equal to each other and

$$
\Delta f_{E: M}=\Delta f_{E: M} \approx 290 \mathrm{MHz}
$$

At this point the former correspondent curves of upperand lower-limit of frequency deviations swap their logical meaning. Based on the type of approximation method, the point in which the equality of (2) and (4) happens, or the $S_{F-\text { e, }}$ varies, but the two curves intersects always. It is believed that while the unwanted effect of unscaled SMA connector increases at higher frequencies, the simulation software also uses its internal mathematical models, such as DjordjevicSarkar model [25], to take the frequency-dependent properties of FR4 substrate into the account. At $S_{F-\text { e. }}$.

Also the SMA connector, as an unscaled element of antenna structure, plays an important role specially and smaller scale-factors, or identically at higher frequencies. For example, as illustrated in Figure 2, the size of the SMA connector in about $\% 78$ in length and $\% 68$ in width of the $\mathrm{A}_{7}$ 's. This means that after soldering the SMA connector, the overall size of the $\mathrm{A}_{7}$ antenna in some directions has roughly doubled. Clearly, use of proposed SMA connector is not suggestible for lesser Scale-factors, in spite of its ability to handle higher frequencies. Figure 7 and 8 illustrates its inherent behavior in absence of any soldered electromagnetic structure. The proposed fluctuating no-load $\mathrm{S}_{11}$ curve shows predictable behavior which could be modeled as

$$
S_{11}(S M A)=-4.410+12.011 \frac{\left(f-2.788 \times 10^{8}\right)}{\left(8.88 \times 10^{9}\right)^{2} \sqrt{\pi / 4 \ln (2)}}
$$

While $f$, as the frequency, should be in Hz. As seen in Figure 8, the SMA connector tends to become more matched at higher frequencies. This is mostly because of its electrical length at those frequencies which become more and more comparable with the effective wavelength of guided and propagated signals. So it starts to radiate efficiently. The fluctuating behavior of $\mathrm{S}_{11}$ for $\mathrm{A}_{6}$ and $\mathrm{A}_{7}$ antennas in Figure 3 illustrates this fact figuratively.

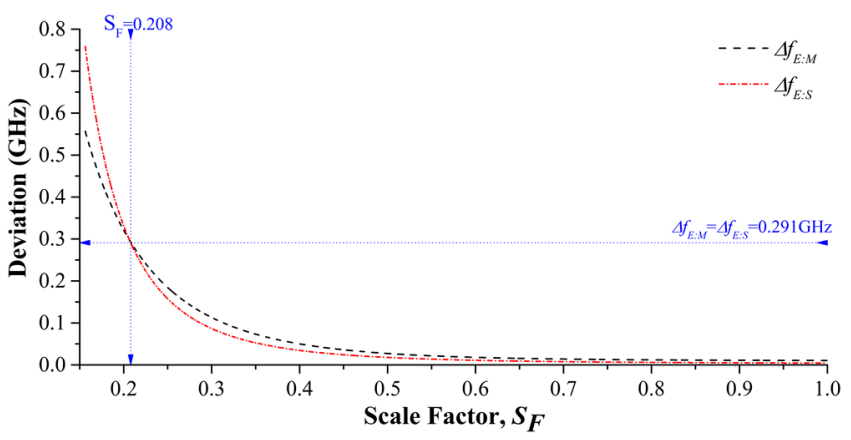

Figure. 9. The Sigmoidal Weibull (type 2) approximations of deviation in measured and simulated resonant frequencies in respect with the theoretical expected values, as describe in (2) and (4).

\section{Application Note}

To apply the findings, some essential hints and assumptions are required. First of all, as the "Substrate Scaling" of microstrip antenna had been studied before[24], this paper has focused on the "Planar Scaling". This means the antenna will be resized but the thickness of substrate and of metallic layer remain constant. This is, mostly, useful when the performance-related parameters of a scaled-up or-down 
version of an embedded antenna is required. To do that, some important issues should be taken into account, which are categorized and explained in Table 2.

Table 2 . The required consideration in scaling process.

\begin{tabular}{|c|c|}
\hline Subject & Consideration(s) \\
\hline $\begin{array}{c}\text { The } \\
\text { Materials }\end{array}$ & $\begin{array}{l}\text { As the superposition property is valid for MEs, they are } \\
\text { considered as a linear system. As described in [4], use of } \\
\text { non-linear or non-homogenous materials, such as Ferrite } \\
\text { and Sapphire respectively, distorts this linearity. On the } \\
\text { other hand it affects the scale-invariability of MEs. }\end{array}$ \\
\hline $\begin{array}{c}\text { The } \\
\text { Substrate }\end{array}$ & $\begin{array}{l}\text { It is highly suggested to select substrate of the original } \\
\text { and scaled antennas form same manufacturer and same } \\
\text { batch of production. This reduces the unwanted } \\
\text { tolerances in } \varepsilon_{r} \text { of the dielectric material, the thickness } \\
\text { and electrical conductivity of the bounded metallic films } \\
\text { on both sides of the substrates. }\end{array}$ \\
\hline $\begin{array}{c}\text { Designed } \\
\text { Electrical } \\
\text { Connections } \\
\end{array}$ & $\begin{array}{l}\text { All of the associated electrical connections, including the } \\
\text { feed-line, directional couplers, filters, etc. should be } \\
\text { scaled too. }\end{array}$ \\
\hline $\begin{array}{l}\text { Standard } \\
\text { Electrical } \\
\text { Connectors/ } \\
\text { Connections }\end{array}$ & $\begin{array}{l}\text { The scaling of standard electrical connectors or } \\
\text { connections is technologically hard to achieve and } \\
\text { financially inefficient. As long as there are an unscaled } \\
\text { connection in electromagnetic structure, it causes some } \\
\text { deviations. }\end{array}$ \\
\hline $\begin{array}{c}\text { Truncation } \\
\text { Error }\end{array}$ & $\begin{array}{l}\text { As described in [12], the truncation error is, potentially, } \\
\text { the source of unwanted error if the rounding procedure } \\
\text { occurs for unsuitable rounding position. The number of } \\
\text { digits which should be retained for measurement and } \\
\text { simulation results are highly dependent on the accuracy } \\
\text { of your measurement device and also the available } \\
\text { computational resources of simulator. }\end{array}$ \\
\hline $\begin{array}{l}\text { Resonant } \\
\text { Frequency } \\
\text { Deviation }\end{array}$ & $\begin{array}{l}\text { For microstrip antenna with soldered SMA connector, the } \\
\text { (2) and (4) hand the upper- and lower-limit of deviations } \\
\text { for various Scale-factors. The measured deviation is } \\
\text { mostly because of frequency-dependent properties of } \\
\text { FR4 substrate and also of the unscaled SMA connector. }\end{array}$ \\
\hline
\end{tabular}

\section{Conclusion}

The Maxwell equations use space-time coordination system and some inherent properties of associated materials of an electromagnetic structure to predict its electrical and magnetic behaviors. So any fluctuation in these parameters along space-time axis affects the measured or calculated results. For example, the nonhomogeneous distribution of electrical permittivity of associated dielectrics results in nonuniform or unpredictable distribution of electrical capacitance along the involved physical axis. The final reactance, which plays significant rule in resonant frequency of electromagnetic structure, will be affected inevitably. This example can be generalized if tolerances in magnetic permeability or physical dimensions to be considered too.

The effects of planar scaling on performance of microstrip patch antenna, fabricated on 1 mm FR4 substrate, were investigated. To reduce the unwanted errors in measurements, calculations and expressing the results, the truncation error was limited to be less than $0.01 \%$. The $A_{7}$ antenna, as the smallest one, showed the best value of $\mathrm{S}_{11}$ magnitude on its probable resonant frequency with $36 \%$ improvement in respect with $\mathrm{A}_{1}$. But some fluctuations were observed in $400 \mathrm{MHz}$ span around resonant frequency of $\mathrm{A}_{6}$ and $A_{7}$ which could be the direct consequence of unscaled SAM connector. The resonant frequency of proposed antennas increases as $S_{F}$ decreases. The measured and simulated resonant frequencies of eight out of nine antennas were shown good convergence to predictions. Lower $S_{F}$ S were associated with larger drift between simulated and measured values in respect with expected resonant frequency, which is as high as $8.3 \%$ or $750 \mathrm{MHz}$ for $\mathrm{A}_{6}$. The constant thickness of the substrate and also frequency dependent value of its dielectric constant are the major sources of drifts. The change rate of the mentioned drift is also smooth, predictable and $S_{F}-$ dependent. The magnitude of measured and simulated $\mathrm{S}_{11}$ also deviated from predicted value which were smooth and predictable as scale-factor decreases, but fluctuating for measured values. In both cases the matching of antenna was enhanced for smaller antennas.

The SMA's frequency dependent characteristics as well as its ability to radiate directly and its unsuitability in scaling applications were shown. The theoretical interpretation of results shed lights on importance of involved standard electrical connectors and handed a simple mathematical model to predict the behavior of selected SMA connector, as well as of upper- and lower limits of frequency deviations. Additionally, to simplify the design procedure and to summarize it for real-world applications, the findings has categorized in tabular form. Despite of all of the above mentioned errors, drifts and tolerances the resonant frequencies showed considerable likeness to predictions of precedent works which validates the results.

\section{References}

[1] J. C. Maxwell, A dynamical theory of the electromagnetic field, Phil. Trans. R. Soc. Lond, vol. 155, p. 459-512, 1865.

[2] N. Hamdan, On the invariance of Maxwell's field equations under Lorentz transformations, Galilean Electrodynamics, vol. 17, p.115-117, 2006.

[3] E. F Knott, Radar cross section measurements, Springer Science and Business Media, Ch. 12, p. 483-510, ISNB: 978-1-4684-9904-9, 2012.

[4] G. Sinclair, Theory of models of electromagnetic systems, In Proceeding of the I.R.E, vol. 36, no. 11, p. 1364-1371, 1948.

[5] F. Comblet, Radar cross section measurements in an anechoic chamber: Description of an experimental system and post processing, IEEE Conference on Antenna Measurements \& Applications, Antibes Juanles-Pins (France), 2014.

[6] J. S. Byrnes, R. Blanchard and F. Capasso, Harvesting renewable energy from Earth's mid-infrared emissions, Proceeding of the national Academy of Sciences of United States of America (PNAS), vol. 111, no. 11, p. 3927-3932, 2014.

[7] P. H. Siegel, Terahertz technology, IEEE Transactions on Microwave Theory and Techniques, vol. 50, no. 3, 2002.

[8] T. S. Cory, Scale-Model Measurements on a Sloping wire Antenna, Research Memorandum 4, Stanford Research Institute, SHI Project 4240-5, Proposed for: United States Army Electronics Research and 
development Laboratory, Sponsored by: ARPA, June 1963.

[9] B. S. Mitchell, An introduction to materials engineering and science for chemical and materials engineers, $1^{\text {st }} \mathrm{ed}$., New Jersey (USA), John Wiley and Sons, Ch. 6, p. 538678, 2004.

[10]E. Fledell, B. Grossman, PCB manufacturing variation impact on high frequency measurement fixtures, $76^{\text {th }}$ ARFTG Microwave Measurement Symposium, Clearwater Beach (USA), p. 1-6, 2010.

[11]L. A. Stefanski, Measurement error models, Journal of the American Statistical Association, vol. 95, no. 452, p. 1353-1358, 2000.

[12] A. Esmaeilkhah, Ch. Ghobadi, J, Nourinia, Upper limit of truncation errors of expressing the real numbers, modeling and the exact solution, $1^{\text {st }}$ National Conference on Modeling Mathematics \& Statistics in Applied Studies, Iran, vol. 1, p. 34-39, 2017.

[13] W. Wu, Y. Zhang and X. Li, Galilean transformation, Lorentz transformation, and a new transformation, International Conference on Transportation, Mechanical, and Electrical Engineering (TMEE), Changchun (China), 2011.

[14]P. B. Seigel, Maxwell's equations under Galilean transformations, $1^{\text {st }}$ ed., San Diego, San Diego State University, 1977.

[15] Ch. S. Pyo, Lorentz transform and Maxwell Equation, $1^{s t}$ Conference on Physical Mathematics, KAIST University, South Korea, 2011.

[16]H. Poincare, La théorie de Lorentz et le principe de reaction, Archives nèerlandaises des Sciences exactes et naturelles, no. 2, vol. 5, p. 252-278, 1900.

[17] A. Einstein, On electrodynamics of moving bodies, Annalen der Physikm, no. 4, vol. 17, pp. 891-921, 1905.

[18]D. H. Schaubert, et al., Effect of microstrip antenna substrate thickness and permittivity, IEEE Transactions on Antennas and Propagation, vol. 37, no. 6, pp. 677682, 1989.

[19]R. Q. Lee, et al., Experimental study of the cross polarization characteristics of rectangular patch antennas, Digest on Antennas and Propagation Society International Symposium, San Jose (USA), vol. 2, pp. 636-639, 1989.

[20] V. Timoshevski, Y. Ke, et al., The influence of surface roughness on electrical conductance of thin $\mathrm{Cu}$ films: $\mathrm{An}$ ab initio study, Journal of Applied Physics, vol. 103, no. 11, 2008.

[21]N. C. Currie, Radar reflectivity measurement: Techniques and applications, $1^{\text {st }}$ ed., Artech House, Norwood (USA), p. 767, 1989.

[22] J, Cech, et al., Surface roughness reduction using spraycoated hydrogen silsesquioxane reflow, Applied Surface Science, vol. 280, pp. 424-430.
[23] J. Goergen, M. Hernandez, A definition of FR-4, IEEE 802 LAN/MAN Standards Committee, IEEE P802.3ap Task Force, 16 Pages [Online].

[24] A. Esmaeilkhah, C. Ghobadi, J. Nourinia, and M. Majidzadeh, "Effect of Substrate Scaling on Microstrip Patch Antenna Performance", AEM, vol. 7, no. 5, pp. 8286, Nov. 2018.

[25] A.R. Djordjevic, T.K. Sarkar, et al, "Wideband frequency-domain characterization of FR-4 and timedomain causality", IEEE Transactions on Electromagnetic Compatibility, vol. 43, no. 4, pp. 662 6672001. 